\title{
A multiscale model for magneto-elastic behaviour including hysteresis effects
}

Received: 10 November 2013 / Accepted: 7 February 2014 / Published online: 17 May 2014

(C) The Author(s) 2014. This article is published with open access at Springerlink.com

\begin{abstract}
Magnetic and mechanical behaviour are strongly coupled: an applied stress modifies the magnetic behaviour, and on the other hand, magnetic materials undergo a magnetisation-induced strain known as the magnetostriction strain. These coupling effects play a significant role on the overall performance of electromagnetic devices such as magnetostrictive transducers or high-performance electric machines. In order to provide engineers with accurate design tools, magneto-elastic effects must be included into constitutive laws for magnetic materials. The origin of the magneto-elastic coupling lies in the competitive contributions of stress and magnetic field to the definition of magnetic domain configurations in magnetic materials. The magnetic domain scale is then suitable to describe magneto-elastic interactions, and this is the reason why multiscale approaches based on a micro-mechanical description of magnetic domain structures have been developed in the last decades. We propose in this paper an extension of a previous anhysteretic multiscale model in order to consider hysteresis effects. This new irreversible model is fully multiaxial and allows the description of typical hysteresis and butterfly loops and the calculation of magnetic losses as a function of external magneto-mechanical loadings. It is notably shown that the use of a configuration demagnetising effect related to the initial domain configuration enables to capture the non-monotony of the effect of stress on the magnetic susceptibility. This configuration demagnetising effect is also relevant to describe the effects of stress on hysteresis losses and coercive field.
\end{abstract}

Keywords Magneto-mechanical couplings - Magnetostriction - Constitutive laws · Micro-mechanical modelling $\cdot$ Hysteresis loops

\section{Introduction}

Ferro- and ferrimagnetic materials are widely used as a basic constituent of electromagnetic devices and transducers. The performance requirements of these devices are strongly increasing with miniaturisation and lightening constraints. As a consequence of these constraints, the magneto-mechanical loadings experienced by magnetic materials are reaching higher intensities (e.g. centrifugal forces in high-speed rotating electrical

L. Daniel ( $\varangle)$

School of Materials, University of Manchester, M13 9PL, Manchester, UK

E-mail: laurent.daniel@u-psud.fr

L. Daniel · M. Rekik

Laboratoire de Génie Electrique de Paris (LGEP), CNRS(UMR8507)/SUPELEC/UPMC/Univ Paris-Sud

11 rue Joliot-Curie, Plateau de Moulon, 91192, Gif-sur-Yvette, France

M.Rekik · O. Hubert

LMT-Cachan, ENS Cachan/CNRS(UMR8535)/UPMC/PRES Universud Paris

61 avenue du Président Wilson, 94235 Cachan, France 

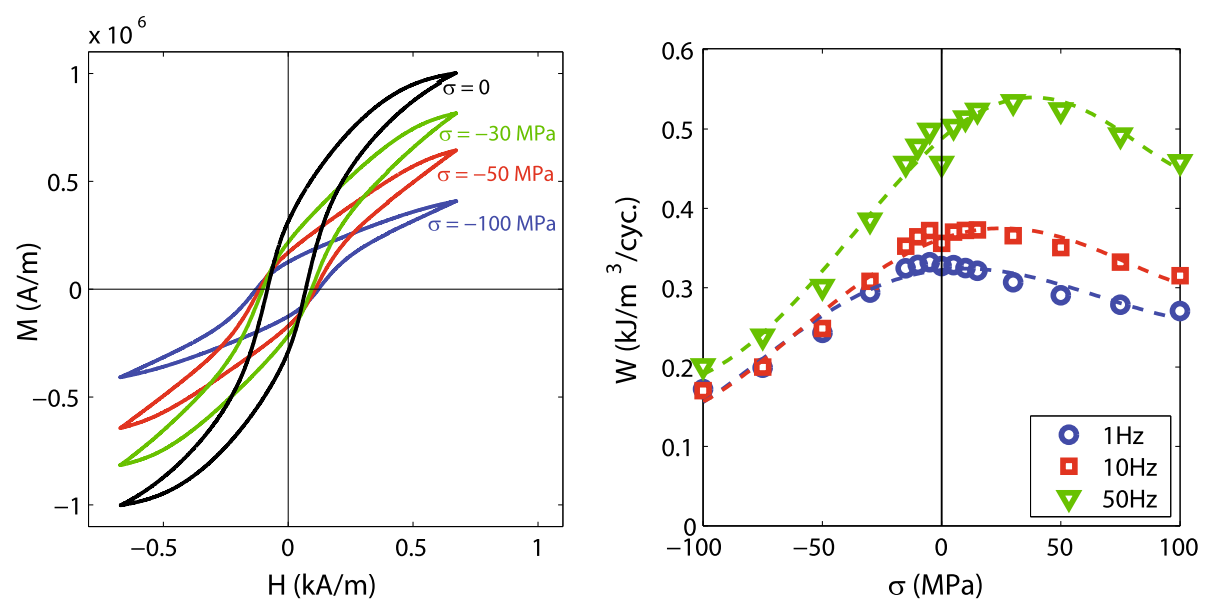

Fig. 1 Experimental illustration of magneto-elastic coupling effects on a non-oriented Iron-Silicon steel: effect of a uniaxial compressive stress on magnetisation at $f=1 \mathrm{~Hz}($ left $)$ and effect of a uniaxial stress on the losses per cycle at different frequencies (right)

machines [1]). The search for materials tailored to specific applications requires the use of advanced constitutive models, able to account for coupled magneto-mechanical phenomena. The magneto-mechanical coupling $[2,3]$ is characterised by the influence of stress on the magnetic susceptibility-explaining the strong effect of stress on the general performance of electromagnetic devices - and by the magnetisation-induced strain, the magnetostriction-exploited in magnetostrictive transducers. Figure 1 illustrates the effect of stress on the magnetic behaviour of a standard non-oriented Iron-Silicon steel (Fe-3\%Si). The left picture shows magnetisation curves under several uniaxial compressive stress (applied in the direction parallel to the magnetic field). It can be seen that stress significantly deteriorates the magnetic permeability and modifies the shape of the hysteresis loop. Hysteresis losses, proportional to the area of hysteresis loops, are also known to be significantly modified by stress [4]. This effect is shown on the right picture where the energy losses per cycle are plotted as a function of stress for several excitation frequencies. It can notably be noticed that the evolution of this energy is non-monotonic with stress intensity. It is recalled that the profile of the losses are highly dependent on the waveform of the excitation field. For the data plotted in Fig. 1, the waveform is triangular with a fixed maximum value for the magnetic field. The results are then expected to be different from standard sinus induction measurements.

The modelling of magneto-mechanical behaviour has been the object of a constant interest over the last century [5]. The theoretical foundations of continuum models for magneto-elasticity have notably been set down by Maugin and Eringen [6-8]. In the last decades, multiscale approaches based on a micro-mechanical description of magnetic domain structures have been developed [9-13] mostly inspired by the early works of Néel [14]. These multiscale approaches seem relevant to establish macroscopic constitutive laws since magneto-elastic coupling effects originates from the evolution of magnetic domain structures under magnetomechanical loading. We propose in this paper to extend such a multiscale scheme in order to describe hysteresis effects and their dependence to magneto-mechanical loadings. In a first part the anhysteretic magnetoelastic model $[12,13]$ used as a basis in this paper will be detailed. The introduction of hysteresis effects will then be explained. After a summary of the modelling approach, numerical results will be discussed and compared to experimental results obtained on an industrial non-oriented Iron-Silicon steel [15]. The discussion will address magnetisation hysteresis loops, magnetostriction butterfly loops and hysteresis losses prediction.

\section{An anhysteretic approach for magneto-elastic couplings}

The model proposed in this paper is derived from a micro-mechanical description of reversible magneto-elastic behaviour $[12,13]$ recalled hereafter. This description relies on the definition of the material free energy at the domain scale and on the use of domains volume fractions as internal variables $[9,10,16]$. It then makes use of scale transition rules to define the behaviour of a polycrystalline representative volume element. 


\subsection{Single-crystal model}

We consider a single crystal $g$. We denote as domain family $\alpha$ the set of domains with magnetisation $\mathbf{M}_{\alpha}$ and magnetostriction strain $\boldsymbol{\varepsilon}_{\alpha}^{\mu}$.

$$
\mathbf{M}_{\alpha}=M_{s} \boldsymbol{\alpha}=M_{s}{ }^{t}\left[\alpha_{1} \alpha_{2} \alpha_{3}\right],
$$

where $\alpha_{i}$ are the direction cosines of the magnetisation $\mathbf{M}_{\alpha}$ and $\boldsymbol{\alpha} . M_{s}$ denotes the saturation magnetisation. In the case of a material with cubic crystallographic symmetry:

$$
\boldsymbol{\varepsilon}_{\alpha}^{\mu}=\frac{3}{2}\left(\begin{array}{ccc}
\lambda_{100}\left(\alpha_{1}^{2}-\frac{1}{3}\right) & \lambda_{111} \alpha_{1} \alpha_{2} & \lambda_{111} \alpha_{1} \alpha_{3} \\
\lambda_{111} \alpha_{1} \alpha_{2} & \lambda_{100}\left(\alpha_{2}^{2}-\frac{1}{3}\right) & \lambda_{111} \alpha_{2} \alpha_{3} \\
\lambda_{111} \alpha_{1} \alpha_{3} & \lambda_{111} \alpha_{2} \alpha_{3} & \lambda_{100}\left(\alpha_{3}^{2}-\frac{1}{3}\right)
\end{array}\right),
$$

where $\lambda_{100}$ and $\lambda_{111}$ are the magnetostriction constants of the material [17]. The free energy $W_{\alpha}$ of a domain $\alpha$ is assumed to be uniform and is the sum of four contributions:

$$
W_{\alpha}=W_{\alpha}^{m a g}+W_{\alpha}^{a n}+W_{\alpha}^{\sigma}+W_{\alpha}^{c o n f} .
$$

$W_{\alpha}^{\text {mag }}$ is the magneto-static energy, tending to align the magnetisation $\mathbf{M}_{\alpha}$ along the magnetic field $\mathbf{H}_{g}$ applied to the single crystal $g$ (Eq. (4)). This equation assumes that the average field over a grain is sufficient to describe the magneto-static contribution (the effect of magnetic field fluctuations within a grain are neglected). $\mu_{0}$ is the vacuum permeability.

$$
W_{\alpha}^{m a g}=-\mu_{0} \mathbf{H}_{g} \cdot \mathbf{M}_{\alpha} .
$$

$W_{\alpha}^{a n}$ is the magneto-crystalline anisotropy energy tending to align the magnetisation along the easy axes. This energetic term explains ${ }^{1}$ the existence of domains microstructure. It is given by Eq.(5) in the case of a cubic system. $K_{1}$ and $K_{2}$ denote the anisotropy constants of the material.

$$
W_{\alpha}^{a n}=K_{1}\left(\alpha_{1}^{2} \alpha_{2}^{2}+\alpha_{2}^{2} \alpha_{3}^{2}+\alpha_{3}^{2} \alpha_{1}^{2}\right)+K_{2}\left(\alpha_{1}^{2} \alpha_{2}^{2} \alpha_{3}^{2}\right) .
$$

$W_{\alpha}^{\sigma}$ is the elastic energy. Assuming uniform strain within a grain, it can be written as a function of the magnetostriction strain $\varepsilon_{\alpha}^{\mu}$ and of the average stress tensor $\sigma_{g}$ in the single crystal $g$ [12]:

$$
W_{\alpha}^{\sigma}=-\sigma_{g}: \varepsilon_{\alpha}^{\mu} .
$$

$W_{\alpha}^{\text {conf }}$ is a configuration term to account for the possible non-randomness of the initial domain configuration (in the absence of applied magneto-mechanical loading) due for instance to plastic deformation [18] or to significant surface effects [19]. This configuration energy can be chosen equivalent to the effect of afictitious-residual $\boldsymbol{\Sigma}^{c}$ stress uniform within the material ${ }^{2}$. In this paper, the configuration residual stress is taken as a uniaxial compressive stress of amplitude $\Sigma^{c}$ along the rolling direction RD.

$$
W_{\alpha}^{\text {conf }}=-\Sigma^{c}: \varepsilon_{\alpha}^{\mu} .
$$

Another significant configuration effect is due to the modification of the initial domain structure when an external stress is applied to the material. Indeed, for some composition of magnetic steels, it has been noticed that the effect of tensile stress on magnetic susceptibility is non-monotonic with increasing stress intensity. This is presumably due to the effect of stress on the initial domain configuration. This effect can be introduced in the configuration term (7), but it is more convenient to consider this stress effect as a configurationfictitious-demagnetising effect. Let's consider the example of a material with positive magnetostriction. Under tensile stress, the number of domains is higher, and they are mostly oriented in a direction close to the tensile direction. Similarly under compression, the number of domains is lower, and they are mostly oriented in a direction perpendicular to the tensile direction. This change in the domain configuration can be translated

\footnotetext{
${ }^{1}$ together with the exchange energy, neglected here because it is uniformly null within any domain family $\alpha$.

2 This configuration effect was modelled thanks to a surface demagnetisation tensor by Hubert and Daniel [19], but it can be shown that the two formulations are equivalent (see Appendix A:).
} 
in terms of demagnetising effect. We then suggest to correct the applied field $\mathbf{H}_{g}$ by a fictitious configuration field $\mathbf{H}_{g}^{\text {conf }}$ depending on the stress state. We propose the following definition for $\mathbf{H}_{g}^{\text {conf }}$ :

$$
\mathbf{H}_{g}^{\text {conf }}=\eta\left(N_{g}-\frac{1}{3}\right) \mathbf{M}_{g},
$$

where $\eta$ is a material parameter, $\mathbf{M}_{g}$ and $N_{g}$ defines the "stress-demagnetisation" effect (Eq.(9)). Its value belongs to the interval [ $\left[\begin{array}{ll}0 & 1\end{array}\right]$ and is $1 / 3$ when no stress is applied:

$$
N_{g}=\frac{1}{1+2 \exp \left(-K \sigma_{g}^{e q}\right)},
$$

where $K$ is a material parameter ${ }^{3}, \sigma_{g}^{e q}$ is the equivalent stress for $\sigma_{g}$ as defined by Daniel and Hubert [20] (namely the projection along the magnetic field direction of the deviatoric part of $\boldsymbol{\sigma}_{g}$ ). $\mathbf{h}$ (unit vector) is the direction of the magnetic field $\mathbf{H}_{g}$.

$$
\sigma_{g}^{e q}=\frac{3}{2} \mathbf{h} \cdot\left(\boldsymbol{\sigma}_{g}-\frac{1}{3} \operatorname{tr}\left(\boldsymbol{\sigma}_{g}\right) \mathbf{I}\right) \cdot \mathbf{h} .
$$

This stress-magnetisation effect was not included in the original magneto-elastic modelling [12]. It is necessary to describe the non-monotonic stress effect on the magnetic susceptibility. The choices made for $\mathbf{H}_{g}^{\text {conf }}$ and $N_{g}$ are more explicitly explained in Appendix B:.

Once the free energy $W_{\alpha}$ is known for a given domain family $\alpha$, the volume fractions $f_{\alpha}$ of domain families $\alpha$ are introduced as internal variables, as already proposed in the literature $[9,10,14,16]$. These internal variables are calculated according to an explicit Boltzmann-type relation [10,12,13]:

$$
f_{\alpha}=\frac{\exp \left(-A_{s} \cdot W_{\alpha}\right)}{\int_{\alpha} \exp \left(-A_{s} \cdot W_{\alpha}\right) \mathrm{d} \alpha}
$$

where $A_{s}$ is an adjustable material parameter ${ }^{4}$. The integration in Eq. (11) is defined over all the possible directions of space for the unit vector $\boldsymbol{\alpha}$. From a practical point of view, it is performed using a discrete mesh of the unit sphere counting 10242 directions [13].

An alternative is the use of Eq. (12) introducing two adjustable parameters $A_{s}$ and $B_{s}$ [21]. It allows to adjust separately the effect of stress and magnetic field on the magnetisation. This separation should not be required if the free energy $W_{\alpha}$ is rigorously written, but the assumptions made-particularly for the elastic energy (see [12])—can lead to significant uncertainties.

$$
f_{\alpha}=\frac{\exp \left(-A_{s} \cdot W_{\alpha}\right)+\exp \left(-A_{s} \cdot W_{-\alpha}\right)}{\int_{\alpha} \exp \left(-A_{s} \cdot W_{\alpha}\right) \mathrm{d} \alpha} \frac{\exp \left(-B_{s} \cdot W_{\alpha}\right)}{\exp \left(-B_{s} \cdot W_{\alpha}\right)+\exp \left(-B_{s} \cdot W_{-\alpha}\right)} .
$$

$W_{-\alpha}$ is the free energy of the domain with magnetisation along $-\alpha$. By choosing $A_{s}=B_{s}$, Eq. (12) reduces to Eq. (11). In the case of Iron-Silicon steels, the use of Eq. (11) is sufficient to describe the magneto-elastic behaviour with satisfactory accuracy; it will thus be retained in the following.

Once the volume fraction is known for any direction $\boldsymbol{\alpha}$ of the magnetisation $\mathbf{M}_{\alpha}$, the magnetisation $\mathbf{M}_{g}$ and the magnetostriction strain $\varepsilon_{g}^{\mu}$ at the single-crystal scale are obtained with a volume average over the single crystal.

$$
\begin{aligned}
\mathbf{M}_{g} & =\left\langle\mathbf{M}_{\alpha}\right\rangle_{g}=\int_{\alpha} f_{\alpha} \mathbf{M}_{\alpha} \mathrm{d} \alpha . \\
\boldsymbol{\varepsilon}_{g}^{\mu} & =\left\langle\boldsymbol{\varepsilon}_{\alpha}^{\mu}\right\rangle_{g}=\int_{\alpha} f_{\alpha} \boldsymbol{\varepsilon}_{\alpha}^{\mu} \mathrm{d} \alpha .
\end{aligned}
$$

\footnotetext{
${ }^{3} K$ can actually be defined as a function of two other modelling parameters, $A_{s}$ and $\lambda_{100}: K \approx \frac{3}{5} A_{s} \lambda_{100}$, see Appendix B:.

${ }^{4} A_{s}$ has been shown to be proportional to the initial slope $\chi^{o}$ of the unstressed anhysteretic magnetisation curve [12]: $A_{s}=3 \chi^{o} / \mu_{0} M_{s}^{2}$.
} 


\subsection{Polycrystal model}

In order to define the behaviour of a polycrystalline representative volume element, the single crystal is incorporated into a self-consistent polycrystalline scheme. This self-consistent approach has been previously detailed [12] and is only briefly recalled hereafter. The local magneto-mechanical loading-local stress $\boldsymbol{\sigma}_{g}$ and magnetic field $\mathbf{H}_{g}$-is derived from the macroscopic magneto-mechanical loading-macroscopic stress $\sigma_{m}$ and magnetic field $\mathbf{H}_{m}$ - using the localisation Eqs. (15) and (16).

$$
\begin{aligned}
\boldsymbol{\sigma}_{g} & =\mathcal{B}_{g}^{\sigma}: \boldsymbol{\sigma}_{m}+\mathcal{L}_{g}^{\sigma}:\left(\boldsymbol{\varepsilon}_{m}^{\mu}-\boldsymbol{\varepsilon}_{g}^{\mu}\right) . \\
\mathbf{H}_{g} & =\mathcal{A}_{g}^{H} \cdot \mathbf{H}_{m}+\mathcal{M}_{g}^{H} \cdot\left(\mathbf{M}_{m}-\mathbf{M}_{g}\right) .
\end{aligned}
$$

$\mathcal{B}_{g}^{\sigma}$ and $\mathcal{A}_{g}^{H}$ are the elastic and magnetic localisation operators and $\mathcal{L}_{g}^{\sigma}$ and $\mathcal{M}_{g}^{H}$ are the elastic and magnetic incompatibility tensors defining the incompatibilities raised by the difference of behaviour between an individual grain and the surrounding medium. These tensors depend on the crystallographic orientation of the considered grain so that texture effects can be included in the modelling (see for instance [19]). The practical calculation of the localisation operators is detailed in ref. [12] and [22] and is recalled in Appendix C:. They notably depend on the elastic stiffness coefficients $C_{\mathrm{ij}}$ of the single crystal. Equation (15) and (16) make use of the macroscopic magnetostriction strain $\boldsymbol{\varepsilon}_{m}^{\mu}$ and magnetisation $\mathbf{M}_{m}$ so that the scheme is self-consistent. Once the local magneto-mechanical loading $\left(\boldsymbol{\sigma}_{g}, \mathbf{H}_{g}\right)$ is known, the single-crystal model is applied to obtain the strain and magnetisation at the single-crystal scale. The macroscopic magnetisation $\mathbf{M}_{m}$ and magnetostriction strain $\boldsymbol{\varepsilon}_{m}^{\mu}$ are then obtained with a volume average over the polycrystal.

$$
\begin{aligned}
\mathbf{M}_{m} & =\left\langle{ }^{t} \mathcal{A}_{g}^{H} \cdot \mathbf{M}_{g}\right\rangle_{m} . \\
\boldsymbol{\varepsilon}_{m}^{\mu} & =\left\langle{ }^{t} \mathcal{B}_{g}^{\sigma}: \boldsymbol{\varepsilon}_{g}^{\mu}\right\rangle_{m} .
\end{aligned}
$$

The macroscopic elastic strain $\boldsymbol{\varepsilon}_{m}^{e l}$ (obtained using the standard macroscopic Hooke law) can be added to the magnetostriction strain to obtain the total macroscopic strain $\boldsymbol{\varepsilon}_{m}$.

\section{Hysteresis effects}

The proposed multiscale approach is anhysteretic so far-it is restricted to the reversible part of magnetoelastic behaviour. Although it captures the complex coupling between elastic and magnetic effects, it does not include dissipation phenomena that are key to the understanding of electromagnetic devices. In the following, we propose to introduce hysteresis effects in the multiscale model. The dissipation is introduced in the singlecrystal model by adding an irreversible contribution $\mathbf{H}_{g}^{i r r}$ to the anhysteretic magnetic field. The definition of $\mathbf{H}_{g}^{i r r}$ is based on the works by Hauser [23], extended to magneto-mechanical loadings:

$$
\left\|\mathbf{H}_{g}^{i r r}\right\|=\delta\left(\frac{k_{r}}{\mu_{0} M_{s}}+c_{r}\left\|\mathbf{H}_{g}\right\|\right)\left[1-\kappa_{g} \exp \left(-\frac{k_{a}}{\kappa_{g}}\left\|\mathbf{M}_{g}-\mathbf{M}_{g}^{r e b}\right\|\right)\right] .
$$

$\mathbf{H}_{g}^{i r r}$ is assumed to be parallel to $\mathbf{H}_{g} . \delta$ is equal to \pm 1 , depending on whether the material is being loaded or unloaded. The sign of $\delta$ starts as positive and is then changed each time there is an inversion in the loading direction. $k_{r}, c_{r}, k_{a}$ and $\kappa_{g}$ are material parameters. The value of $\kappa_{g}$ changes each time there is an inversion in the loading direction. The new value $\kappa_{g}$ is calculated from the previous value $\kappa_{g}^{o}$ according to Eq. (20). The initial value $\kappa_{g}^{i n i}$ of $\kappa_{g}$ is a material constant. $\mathbf{M}_{g}^{r e b}$ is the value of $\mathbf{M}_{g}$ at the previous inversion of the loading direction.

$$
\kappa_{g}=2-\kappa_{g}^{o} \exp \left(-\frac{k_{a}}{\kappa_{g}^{o}}\left\|\mathbf{M}_{g}-\mathbf{M}_{g}^{r e b}\right\|\right) .
$$

In the case of a purely magnetic loading, an inversion of loading direction is defined as a change of sign for the time derivative of the applied magnetic field. From a practical point of view, this inversion is detected at instant $t$ when $\Delta \mathbf{H}_{m}^{t} \cdot \Delta \mathbf{H}_{m}^{t-1}<0$. In the case of a purely mechanical loading, an inversion of loading direction is defined as a change of sign for the time derivative of the applied stress. From a practical point of view, 


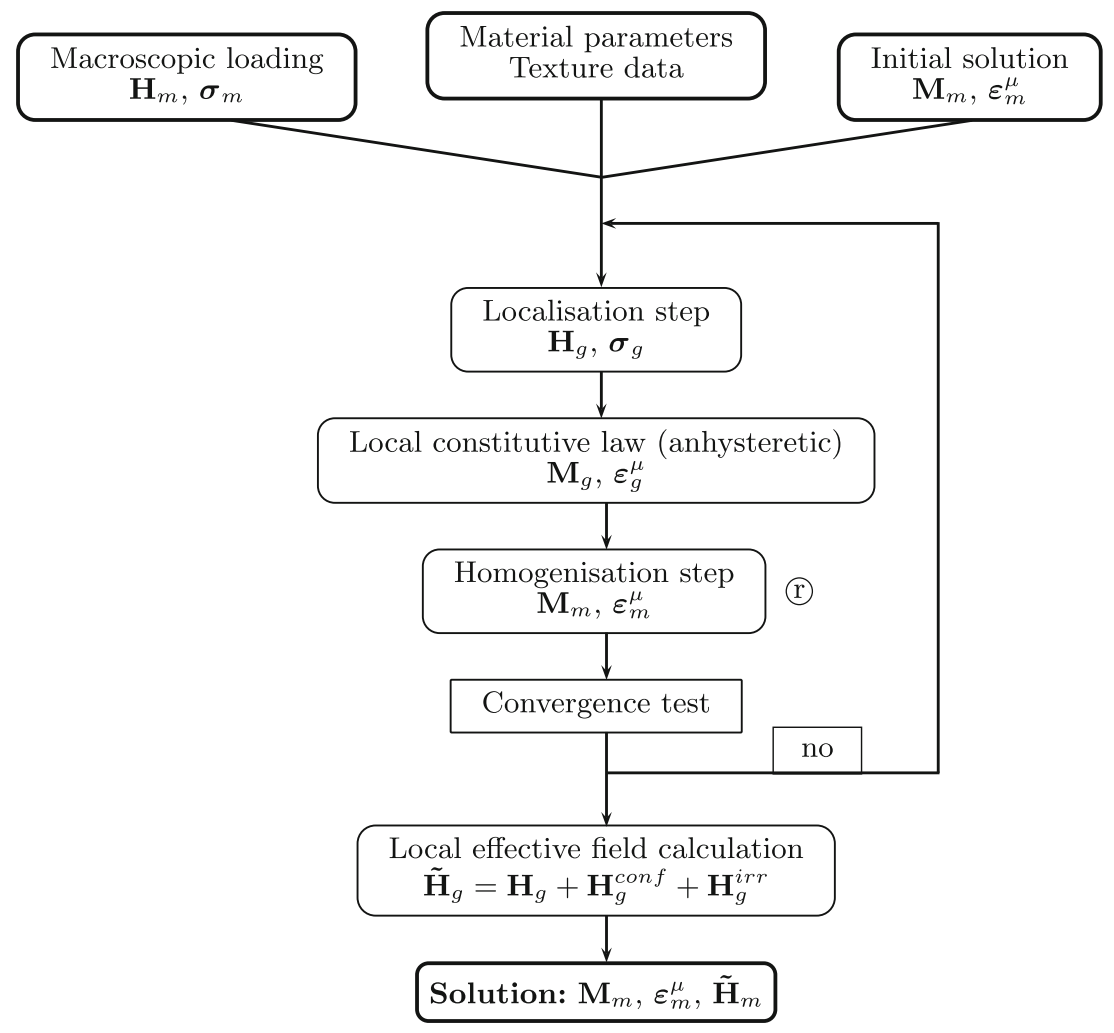

Fig. 2 Calculation principle

this inversion is detected at instant $t$ when $\Delta \boldsymbol{\sigma}_{m}^{t}: \Delta \boldsymbol{\sigma}_{m}^{t-1}<0$. More generally, an inversion of magnetomechanical loading direction in a grain $g$ can be defined at instant $t$ using the average free energy $W_{g}$ of grain $g\left(W_{g}=\left\langle W_{\alpha}\right\rangle_{g}\right)$ :

$$
\Delta W_{g}^{t} \cdot \Delta W_{g}^{t-1}<0 .
$$

In order to account for the dependence of the coercive field to the applied stress, the parameter $k_{r}$ - defining the coercive field - is assumed to show a dependence to stress similar to the stress configuration effect introduced in section $2.1, k_{r}^{0}$ being a material constant:

$$
k_{r}=k_{r}^{0}\left(\frac{4}{3}-N_{g}\right) .
$$

\section{Modelling summary}

The modelling process can be summarised as presented in Fig.2.

The input data are the material parameters (including the crystallographic texture) and the applied macroscopic loading in terms of stress $\boldsymbol{\sigma}_{m}$ and magnetic field $\mathbf{H}_{m}$. An initial guess for the solution (macroscopic magnetisation $\mathbf{M}_{m}$ and magnetostriction strain $\boldsymbol{\varepsilon}_{m}^{\mu}$ ) is also needed to start the process. The use of the solution under uniform stress and uniform magnetic field assumptions is usually convenient for this initial guess. For each element of the orientation distribution function, the localisation rules (15) and (16) are applied to define the local stress $\boldsymbol{\sigma}_{g}$ and magnetic field $\mathbf{H}_{g}$. The local anhysteretic constitutive law is then applied according to the model presented in section 2.1 to obtain the local magnetisation $\mathbf{M}_{g}$ and magnetostriction strain $\boldsymbol{\varepsilon}_{g}^{\mu}$. These local responses are then averaged over the crystallographic orientations to obtain the macroscopic magnetisation $\mathbf{M}_{m}$ and magnetostriction $\boldsymbol{\varepsilon}_{m}^{\mu}$ (Eqs. (17) and (18)). This solution replaces the initial guess, and the process is repeated until convergence. In order to ensure the convergence, a relaxation method can be used just after 
Table 1 Material parameters: reversible model

\begin{tabular}{llllllll}
\hline Parameter & $M_{S}$ & $\left(K_{1}, K_{2}\right)$ & $\left(\lambda_{100}, \lambda_{111}\right)$ & $\left(C_{11}, C_{12}, C_{44}\right)$ & $A_{s}$ & $\Sigma^{c}$ & $\eta$ \\
\hline Value & $1.6110^{6}$ & $(38,0)$ & $(23,-4.5)$ & $(202,122,229)$ & $310^{-3}$ & 20 & $210^{-4}$ \\
Unit & $\mathrm{A} / \mathrm{m}$ & $\mathrm{kJ} / \mathrm{m}^{3}$ & $10^{-6}$ & $\mathrm{GPa}$ & $\mathrm{m}^{3} / \mathrm{J}$ & $\mathrm{MPa}$ & - \\
\hline
\end{tabular}

Table 2 Material parameters: local effective field

\begin{tabular}{llllc}
\hline Parameter & $k_{r}^{0}$ & $c_{r}$ & $k_{a}$ & $\kappa_{g}^{\text {ini }}$ \\
\hline Value & 150 & 0.1 & $1510^{-6}$ & 1 \\
Unit & $\mathrm{J} / \mathrm{m}^{3}$ & - & $\mathrm{m} / \mathrm{A}$ & - \\
\hline
\end{tabular}

the homogenisation step. This process, noted by the symbol $\mathbb{P}$ in Fig. 2, consists in defining the new value of $\mathbf{M}_{m}$ and $\boldsymbol{\varepsilon}_{m}^{\mu}$ at step $k$ as a weighted average between the result of the homogenisation step and the previous result at step $k-1$. The weighting coefficient has been set to 0.5 for the computations presented in this paper. When convergence is reached, the local magnetic field $\mathbf{H}_{g}$ for each crystallographic orientation is corrected by $\mathbf{H}_{g}^{\text {conf }}$ (Eq. (8)) and $\mathbf{H}_{g}^{\text {irr }}$ (Eq. (19)) to obtain the local effective field $\tilde{\mathbf{H}}_{g}$ accounting for the stress configuration effect and the hysteresis losses:

$$
\tilde{\mathbf{H}}_{g}=\mathbf{H}_{g}+\mathbf{H}_{g}^{\text {conf }}+\mathbf{H}_{g}^{i r r} .
$$

The final solution is given by the macroscopic magnetisation $\mathbf{M}_{m}$, the macroscopic magnetostriction strain $\boldsymbol{\varepsilon}_{m}^{\mu}$ and the average magnetic field $\tilde{\mathbf{H}}_{m}$ :

$$
\tilde{\mathbf{H}}_{m}=\left\langle\tilde{\mathbf{H}}_{g}\right\rangle_{m} .
$$

\section{Modelling results and comparison to experiments (1D)}

The proposed multiscale approach has been applied to model the behaviour of a commercial non-oriented $3 \% \mathrm{Si}-\mathrm{Fe}$ steel from Arcelormittal delivered in $0.5-\mathrm{mm}$ thick sheets. It is a standard material for rotating machines. A comprehensive experimental characterisation for this material can be found in Rekik et al. [15].

\subsection{Material parameters}

The material parameters used for the modelling are given in Table 1 for the anhysteretic part of the behaviour and in Table 2 for the dissipative part. A brief guide to the identification of these parameters is given in Appendix D:.

The crystallographic texture data consists in a set of 396 crystallographic orientations representative for this material and obtained using electron back scattered diffraction (EBSD). The corresponding discrete pole figures are plotted in Fig. 3.

\subsection{Anhysteretic behaviour}

The application of the anhysteretic constitutive model is presented in Figs. 4, 5, 6, 7 and 8 in the case of a nonoriented 3\% Iron-Silicon alloy. Figures 4 and 5 describe the anisotropy of the magneto-elastic behaviour of the material in the absence of applied stress. $\mathrm{RD}, \mathrm{TD}$ and $45^{\circ}$ denote the rolling direction, the transverse direction and a direction at $45^{\circ}$, respectively. The experimental results are consistent with earlier measurements [24]. In the modelling, anisotropy effects are introduced through the measured crystallographic texture and with the parameter $\boldsymbol{\Sigma}^{c}$ (Eq. (7)). The latter is chosen so that the magnetostrictive anisotropy is correctly described at saturation (Fig. 5).

As already noticed by Daniel et al. [12], the model tends to overestimate the magnetic susceptibility (Fig. 4). This is attributed to the approximations in the definition of the free energy and particularly the fact that intragranular heterogeneities and local demagnetising effects are insufficiently taken into account. The 


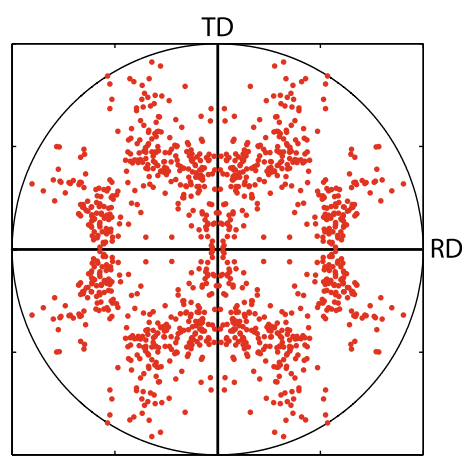

$<100>$ poles

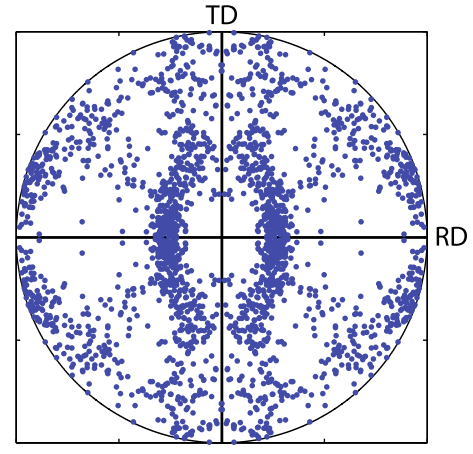

$<110>$ poles

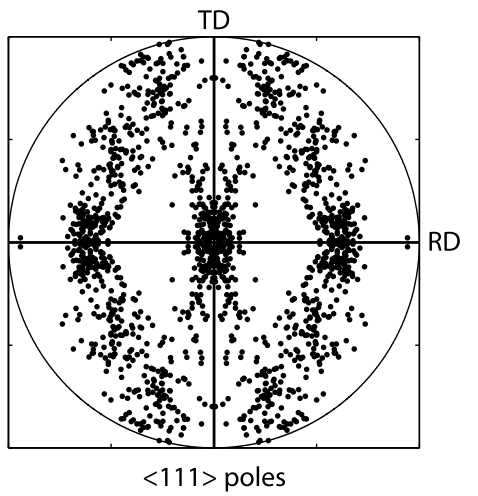

Fig. 3 Discrete pole figures (396 orientations) for a non-oriented $3 \% \mathrm{Fe}-\mathrm{Si}$ alloy
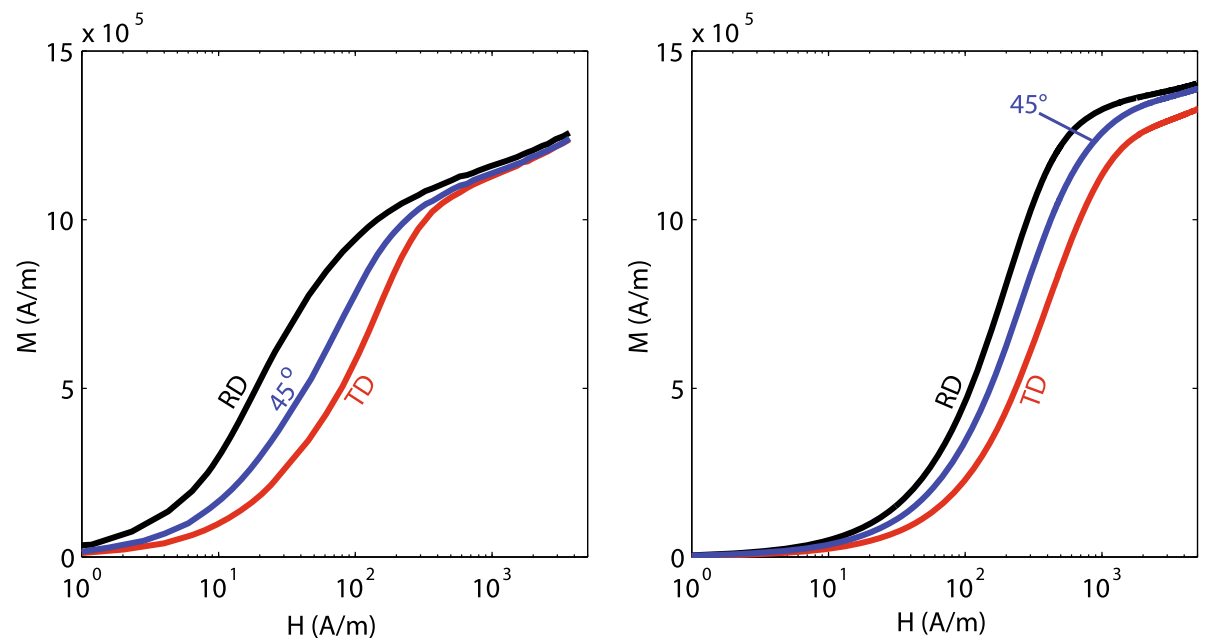

Fig. 4 Anisotropy of the anhysteretic magnetic behaviour of a non-oriented Iron-Silicon steel: magnetisation curves along rolling, transverse and $45^{\circ}$ directions. Experimental measurements (left) and modelling results (right)
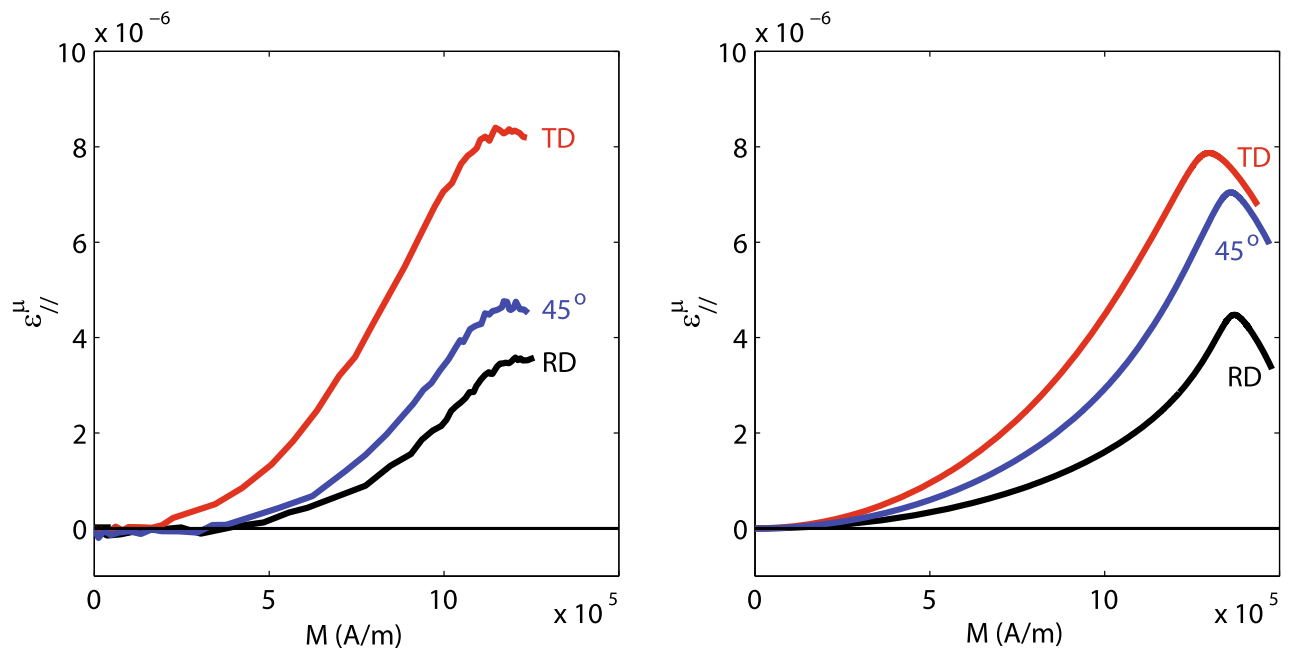

Fig. 5 Anisotropy of the anhysteretic magnetostrictive behaviour of a non-oriented Iron-Silicon steel: magnetostriction curves along rolling, transverse and $45^{\circ}$ directions. Experimental measurements (left) and modelling results (right) 

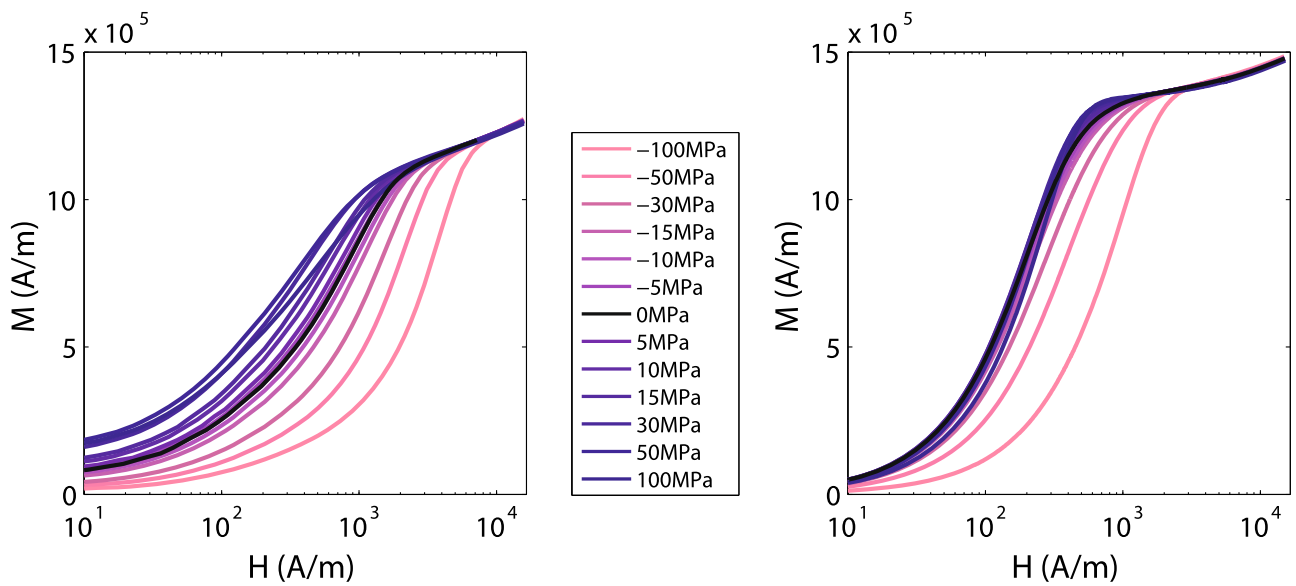

Fig. 6 Anhysteretic magneto-elastic behaviour of a non-oriented Iron-Silicon steel along the rolling direction: effect of a uniaxial stress on magnetisation. Experimental measurements (left) and modelling results (right)
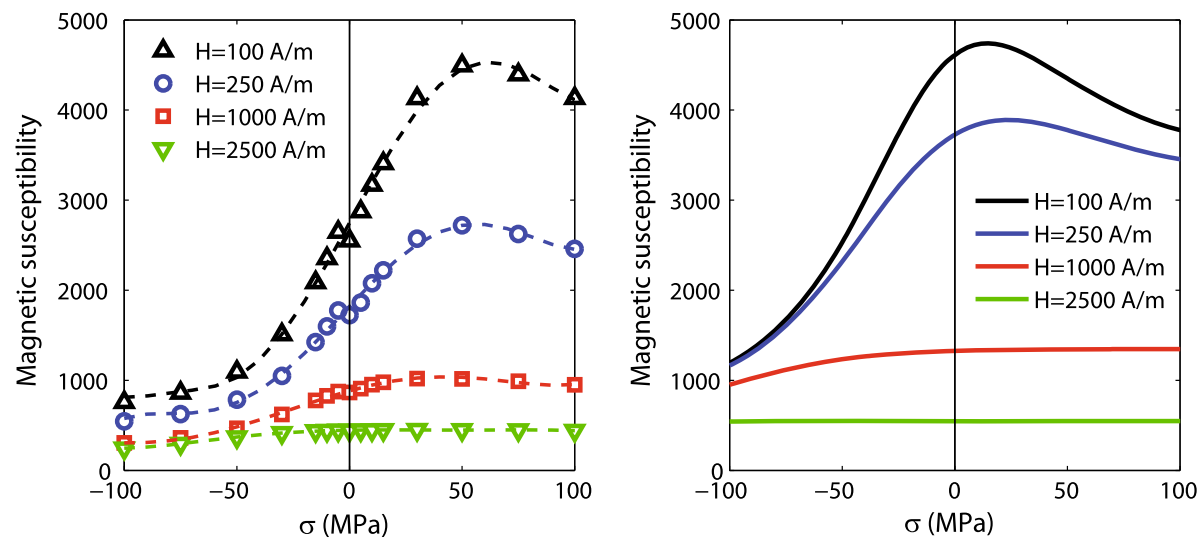

Fig. 7 Anhysteretic secant magnetic susceptibility a non-oriented Iron-Silicon steel along the rolling direction: effect of a uniaxial stress. Experimental measurements (left) and modelling results (right)

general trends, however, and the anisotropy effects are correctly rendered by the model. The description of the magnetostriction strain is very satisfactory (Fig. 5), notably due to the use of the parameter $\boldsymbol{\Sigma}^{c}$, although the longitudinal strain when the magnetic field is applied at $45^{\circ}$ from the rolling direction is slightly overestimated.

Figure 6 shows the magnetic behaviour of the material submitted to a uniaxial stress applied along the rolling direction (RD). The magnetic field is also applied along RD. The overestimation of the magnetisation for a given magnetic field can still be noticed, but the effect of stress on the behaviour is satisfactorily described. A compression leads to a very significant drop in the magnetic susceptibility, whereas a tension has a much slighter effect.

Another way of describing the effect of stress on the magnetic behaviour is to plot the secant magnetic susceptibility as a function of the uniaxial stress intensity. The secant magnetic susceptibility is defined here as the ratio between the magnetisation-measured by its projection along the applied field direction - and the norm of the applied field. The result is shown in Fig. 7. Again the magnetic susceptibility tends to be overestimated but the effect of stress on the magnetic behaviour is appropriately described. Compression is associated to a strong degradation, and tension first increases the magnetic susceptibility but then decreases it. This non-monotonic dependence of the magnetic susceptibility to the applied uniaxial stress is described by the model through the use of the configuration field $\mathbf{H}_{g}^{\text {conf }}$ (Eq. (8)).

The magnetostrictive behaviour under uniaxial stress is shown in Fig. 8. A tensile stress tends to decrease the magnetostriction strain amplitude, whereas a compression tends to increase it. The figures have been plotted so that all the curves start from zero. The $\Delta \mathrm{E}$ effect-associated to the stress-induced magnetostriction strain in the absence of magnetic field [25]—is then not explicitly shown in Fig. 8. The proposed model gives a very satisfactory description of the magnetostrictive behaviour under stress. 

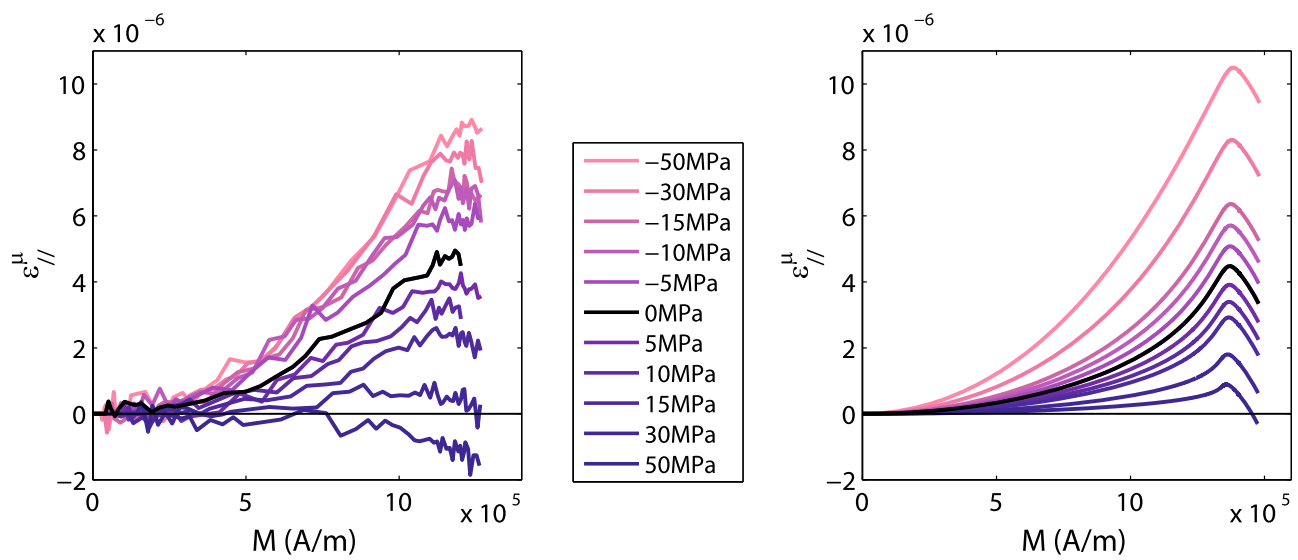

Fig. 8 Anhysteretic magneto-elastic behaviour of a non-oriented Iron-Silicon steel along the rolling direction: effect of a uniaxial stress on magnetostriction strain. Experimental measurements (left) and modelling results (right)
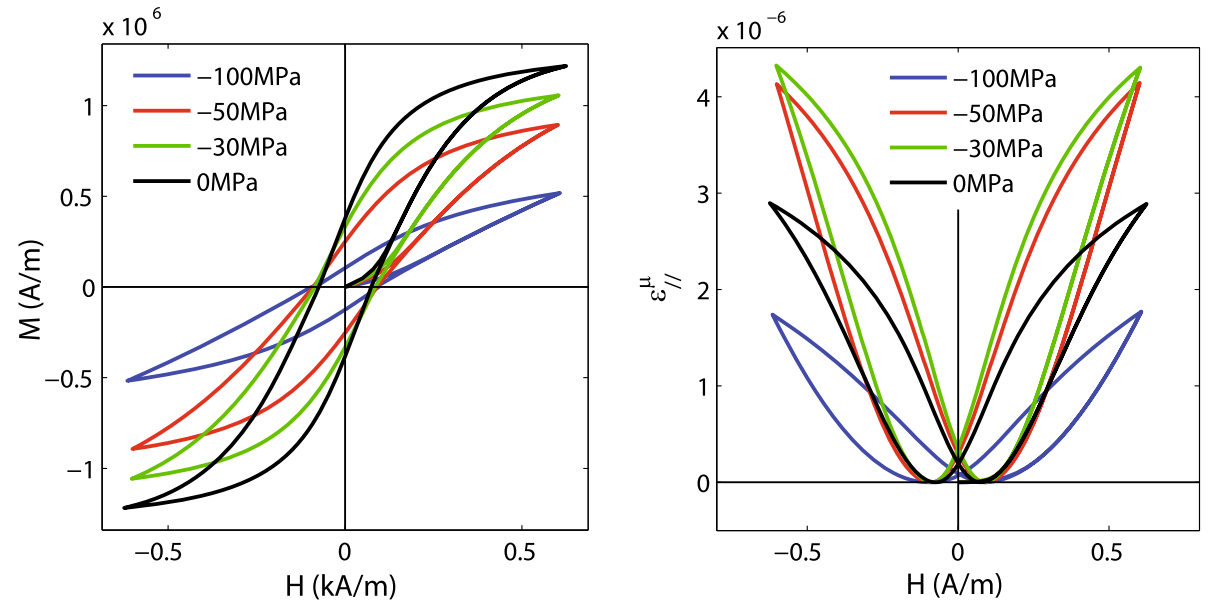

Fig. 9 Magnetisation (left) and magnetostriction (right) loops under uniaxial stress for a non-oriented Iron-Silicon steel loaded along the rolling direction

\subsection{Dissipative behaviour}

The description of hysteresis effects described in section 3 allows calculating the dissipative behaviour of the material under the same magneto-mechanical conditions. Figure 9 shows the predicted hysteresis magnetisation loops under uniaxial stress. These loops very satisfactorily compare to the measured curves presented in Fig. 1(left). Figure 9 shows the corresponding predicted magnetostriction butterfly loops. Due to the very small amplitude of the strains, dynamic measurements could not be performed on this material to compare with these results-only anhysteretic magnetostriction measurements could be carried out [15]. It can be noticed that, for a fixed magnetic field value, the evolution of the magnetostriction strain with respect to stress is not monotonic.

The calculation of the surface of the magnetisation hysteresis loops gives an insight into the effect of stress on magnetic losses. The results are presented in Fig. 10. They are in good agreement with the experimental measurements at low frequency shown in Fig. 1. Both compression and tension tend to decrease the hysteresis losses before a saturation point is reached. It is recalled that this calculation has been performed for a fixed maximum value of the magnetic field. These results cannot be straightforwardly compared to measurements under sinusoidal magnetic inductions.

\section{Conclusion}

A micro-mechanical framework for the description of the magneto-elastic behaviour of polycrystalline magnetic materials has been presented. This multiscale approach is based on an energy description of magneto- 


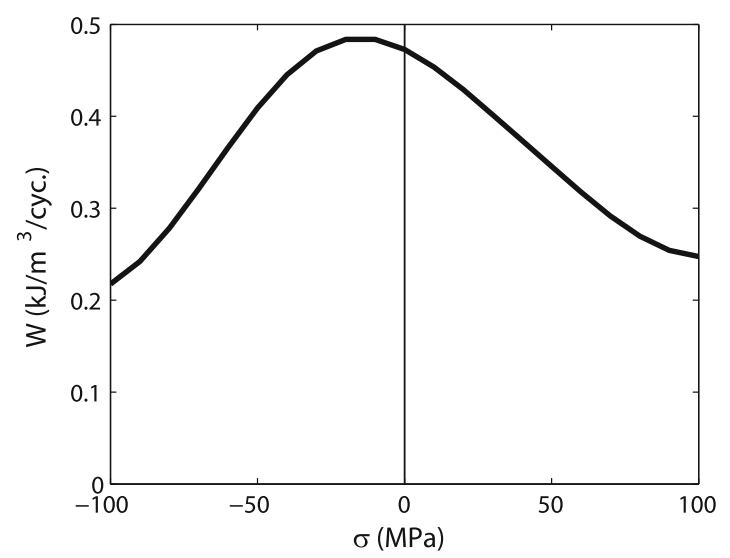

Fig. 10 Prediction of hysteresis losses as a function of the applied uniaxial stress for a non-oriented Iron-Silicon steel

elastic couplings at the magnetic domain scale. It is combined with scale transition rules to describe the macroscopic response of polycrystalline media. Its fully multiaxial definition allows the application of the model in any magneto-mechanical loading configuration, whatever the form of the stress tensor or the relative orientation between stress and magnetic field. It has been used to describe the magnetostriction and magnetisation curves of a magnetic material subjected to uniaxial magneto-mechanical loadings, but it can also be applied to predict piezomagnetic behaviour [26] or $\Delta \mathrm{E}$ effect $[16,25]$. The model accounts for the anisotropy effects at the grain scale - through single-crystal elastic, magnetic and magnetostrictive anisotropy — and at the macroscopic scale-through crystallographic texture. It also accounts for additional anisotropy effects such as surface effects arising when the grain size is large compared to a sample dimension (typically the width of electrical steel sheets). Moreover, the proposed extension to hysteretic behaviour enables to monitor the effect of stress on coercive field and hysteresis losses. The approach is predictive in the sense that it can be applied to a large variety of magneto-mechanical configurations, while the parameters are identified only from a limited number of experimental measurements. The validation results have been limited to uniaxial loading configurations in this paper. However the formulation of the model is fully multiaxial and the confrontation of the predicted behaviour to experimental data obtained under multiaxial loadings will be the object of further communication. The extension of the model for magnetic loadings at higher frequency will also be investigated.

This modelling approach is a promising tool for material design. It can be used to find optimal microstructure (e.g. crystallographic texture, crystal properties) for a given application. This optimisation can be performed on uncoupled mechanical or magnetic properties - as usually done - but can also include magneto-elastic effects. The latter are key for instance to the design of high-speed rotating machines or magnetostrictive actuators. The model can also be used to define appropriate initial domain configurations for specific applications and help to act on external or internal stresses - for instance through assembling, packaging or manufacturing constraints—in order to enhance the performance of existing devices.

Acknowledgments This work was supported by the Agence Nationale de la Recherche under the grant MAEL (BLAN082_367373) and by the Fonds unique interministériel under the grant FUI-OSEO 3MT.

Open Access This article is distributed under the terms of the Creative Commons Attribution License which permits any use, distribution, and reproduction in any medium, provided the original author(s) and the source are credited.

\section{Appendix A: Correspondance between demagnetising surface effect and initial stress configuration term}

This appendix aims at proving the equivalence between the formulation of "surface effect" proposed by Hubert and Daniel [19] and the "configuration term" used in this paper to describe the initial domain configuration in magnetic materials in the absence of applied loading.

In the proposed multiscale modelling, the configuration term (7) has been included in the description of the domains energy balance in order to account for phenomena such as the strong differences between magnetostriction amplitude along the rolling and transverse direction of electrical sheets. The configuration energy is reminded in Eq. (25) where $\boldsymbol{\Sigma}^{c}$ is the fictitious configuration stress tensor. In the following, $\boldsymbol{\Sigma}^{c}$ is assumed to 
be diagonal. A similar approach was already proposed for the modelling of grain-oriented (GO) Iron-Silicon steels [19]. The configuration effect was introduced as a surface energy related to the large ratio between grain size and thickness of the sheets (Eq. (26)). $C$ is a material parameter, $\boldsymbol{\alpha}$ is the direction of the magnetisation (unit vector) and $N^{S}$ is a diagonal second-order tensor introducing the ratio between the average grain size and the dimensions of the specimen (see [19] for details). The grounds for this latter definition is acceptable for GO steel sheets but may not be appropriate for non-oriented Iron-Silicon steels or other materials with small grain size compared to the sheet thickness.

$$
\begin{aligned}
W_{\alpha}^{\text {conf }} & =-\boldsymbol{\Sigma}^{c}: \boldsymbol{\varepsilon}_{\alpha}^{\mu} . \\
W_{\alpha}^{S} & =C \boldsymbol{\alpha} . \boldsymbol{N}^{S} \cdot \boldsymbol{\alpha} .
\end{aligned}
$$

The object of this appendix is to demonstrate that the two formulations (25) and (26) are equivalent. For that purpose, we seek a configuration stress $\Sigma^{c}$ such that the configuration energy (25) is equal to the surface energy (26) except for a constant $C_{0}$ :

$$
-\boldsymbol{\Sigma}^{c}: \boldsymbol{\varepsilon}_{\alpha}^{\mu}=C \boldsymbol{\alpha} \cdot \boldsymbol{N}^{S} \cdot \boldsymbol{\alpha}+C_{0} .
$$

If we assume no magnetisation rotation inside the domains, the magnetostriction strain tensor only depends on $\lambda_{100}$ and can be written:

$$
\boldsymbol{\varepsilon}_{\alpha}^{\mu}=\frac{3}{2} \lambda_{100}\left(\boldsymbol{\alpha} \otimes \boldsymbol{\alpha}-\frac{1}{3} \mathbf{I}\right) .
$$

The configuration energy (25) is then given by:

$$
W_{\alpha}^{\text {conf }}=-\frac{3}{2} \lambda_{100}\left(\Sigma_{11}^{c}\left(\alpha_{1}^{2}-1 / 3\right)+\Sigma_{22}^{c}\left(\alpha_{2}^{2}-1 / 3\right)+\Sigma_{33}^{c}\left(\alpha_{3}^{2}-1 / 3\right)\right) .
$$

On the other hand, the surface energy (27) is given by:

$$
W_{\alpha}^{S}=C\left(N_{11}^{S} \alpha_{1}^{2}+N_{22}^{S} \alpha_{2}^{2}+N_{33}^{S} \alpha_{3}^{2}\right) .
$$

In order to fulfil Eq. (27), the configuration stress $\Sigma^{c}$ can be chosen as:

$$
\Sigma^{c}=-\frac{2 C}{3 \lambda_{100}} N^{S} .
$$

Besides, the constant $C_{0}$ is defined by:

$$
C_{0}=\frac{\lambda_{100}}{2} \operatorname{tr}\left(\Sigma^{c}\right)
$$

$C_{0}$, however, is not used in practice since the free energy (3) is defined except for a constant.

Hence, it is shown that, given this relationship (31) between $\Sigma^{c}$ and $N^{S}$, the two formulations (25) and (26) are equivalent and can be used indifferently.

\section{Appendix B: Stress configuration effect}

The effect of a tensile stress (parallel to the applied magnetic field) on the magnetic susceptibility is nonmonotonic with stress intensity. This experimental observation is in contradiction with the classical magnetoelastic effect described by the magneto-elastic term (6). A complementary effect is then required. It has been introduced in the multiscale modelling through a configuration term (9). The origin of this term is explained in this appendix.

The configuration demagnetising field (8) is defined after the simplified description of the modification of domain structures when an external stress is applied. Let's consider a material with positive magnetostriction coefficient $\lambda_{100}$. The magnetisation rotation mechanism is neglected so that $\lambda_{111}$ is not considered. Under uniaxial tension, the volume fraction of domains oriented along the direction of the applied stress increases. This leads to a higher local demagnetising field that explains the refinement of the domain structure. Under uniaxial compression, the effect is opposite, leading to a decrease of the local demagnetising effect and hence 
to a growth of domain size. Figure 1 gives an illustration for a very schematic domain distribution inside a single crystal. Figure 12 gives a similar illustration for a biaxial stress configuration. The narrowing and growth of domain size occur as well, but for a higher proportion of domains. Demagnetising fields are expected to be reduced compared to the uniaxial configuration since closure paths remain in large proportion. The interpretation of a configuration with a uniaxial loading rotated at $45^{\circ}$ with respect to the main axes (Fig. 13) leads to the same type of distribution, underlining the fact that shear stress has a minor role on the domain distribution and consequently on the configuration demagnetising field.

These schematic illustrations show that there is a strong correlation between domain fractions and demagnetising fields. The demagnetising field is the same for domains with opposite magnetisation. In order to establish an expression for the configuration demagnetising field $\mathbf{H}_{g}^{\text {conf }}$, the following assumptions are considered:

- the magnetisation rotation due to stress is negligible,

- the demagnetising configuration field $\mathbf{H}_{g}^{\text {conf }}$ can be defined at the grain scale and depends on a demagnetising tensor $\boldsymbol{N}^{d}$, diagonal in the coordinate system corresponding to the local magnetic field $\mathbf{H}_{g}$. It is written:

$$
\mathbf{H}_{g}^{\text {conf }}=\boldsymbol{N}^{d}\left(\boldsymbol{\sigma}_{g}\right) \cdot \mathbf{M}_{g},
$$

- the demagnetising configuration field $\mathbf{H}_{g}^{\text {conf }}$ is supposed to be aligned with the local magnetic field $\mathbf{H}_{g}$,

- for the definition of the configuration field $\mathbf{H}_{g}^{\text {conf }}$, only the magneto-elastic energy $W_{\alpha}^{\sigma}$ (6) is considered in the definition of the volume fractions $f_{\alpha}^{\prime}$ :

$$
f_{\alpha}^{\prime}=\frac{\exp \left(-A_{s} W_{\alpha}^{\sigma}\right)}{\int_{\alpha} \exp \left(-A_{s} W_{\alpha}^{\sigma}\right) \mathrm{d} \alpha},
$$

- the stress demagnetising tensor $N^{d}$ is null in the absence of applied stress:

$$
\boldsymbol{N}^{d}\left(\boldsymbol{\sigma}_{g}=\mathbf{0}\right)=\mathbf{0} .
$$

Considering the previous domain structure (Figs. 11, 12 and 13) under these assumptions, the magnetoelastic energy for the six domain families is simplified into:

$$
\begin{aligned}
& W_{1}^{\sigma}=W_{2}^{\sigma}=-\lambda_{100} \sigma_{\mathrm{xx}}+\frac{\lambda_{100}}{2}\left(\sigma_{\mathrm{yy}}+\sigma_{\mathrm{zz}}\right) \\
& W_{3}^{\sigma}=W_{4}^{\sigma}=-\lambda_{100} \sigma_{\mathrm{yy}}+\frac{\lambda_{100}}{2}\left(\sigma_{\mathrm{xx}}+\sigma_{\mathrm{zz}}\right) \\
& W_{5}^{\sigma}=W_{6}^{\sigma}=-\lambda_{100} \sigma_{\mathrm{zz}}+\frac{\lambda_{100}}{2}\left(\sigma_{\mathrm{xx}}+\sigma_{\mathrm{yy}}\right)
\end{aligned}
$$
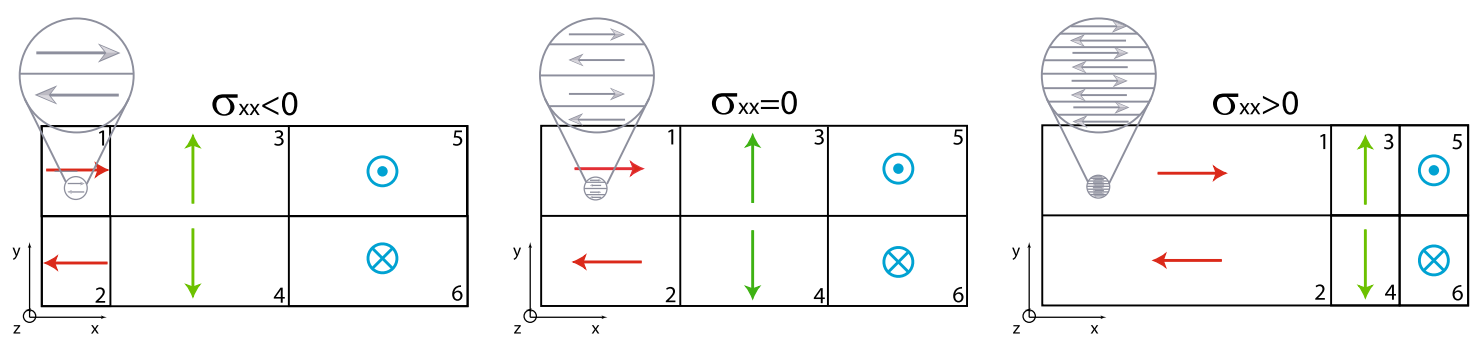

Fig. 11 Evolution of the domain distribution inside a single crystal under uniaxial stress $\sigma_{\mathrm{xx}}$
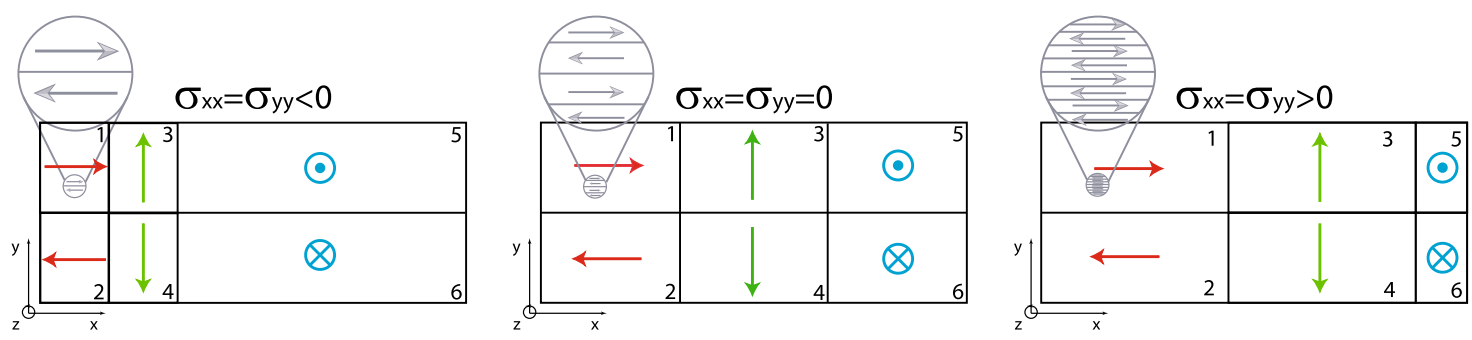

Fig. 12 Evolution of the domain distribution inside a single crystal under equibiaxial stress $\left(\sigma_{\mathrm{xx}}, \sigma_{\mathrm{yy}}\right)$ 

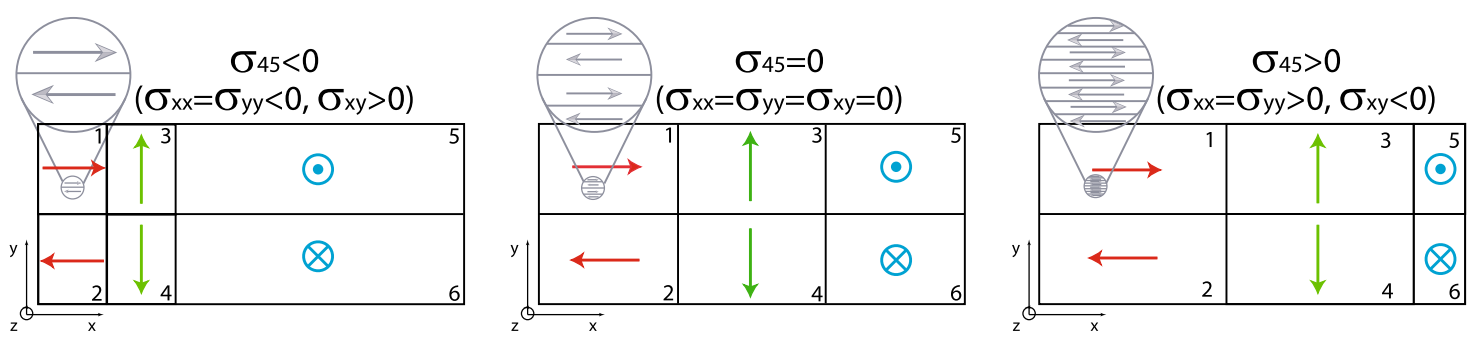

Fig. 13 Evolution of the domain distribution inside a single crystal under uniaxial stress $\sigma_{45}$

The following definition for $\boldsymbol{N}^{d}$ is proposed:

$$
\boldsymbol{N}^{d}=\left(\begin{array}{ccc}
2 f_{1}^{\prime}-1 / 3 & 0 & 0 \\
0 & 2 f_{3}^{\prime}-1 / 3 & 0 \\
0 & 0 & 2 f_{5}^{\prime}-1 / 3
\end{array}\right)_{x y z}
$$

It is easily verified that $\boldsymbol{N}^{d}$ is null when no stress is applied. Considering a magnetic field $\mathbf{H}_{g}$ along $\mathbf{x}$, only the component $N_{\mathrm{xx}}^{d}$ of $N^{d}$ is needed. Using the definition (34) of $f_{1}^{\prime}$, noting $K=\frac{3}{2} A_{s} \lambda_{100}$ and after a few calculations, the demagnetising factor is obtained:

$$
N_{\mathrm{xx}}^{d}=\frac{\exp \left(K \mathbf{x} \cdot \mathbf{s}_{g} \cdot \mathbf{x}\right)}{\exp \left(K \mathbf{x} \cdot \mathbf{s}_{g} \cdot \mathbf{x}\right)+\exp \left(K \mathbf{y} \cdot \mathbf{s}_{g} \cdot \mathbf{y}\right)+\exp \left(K \mathbf{z} \cdot \mathbf{s}_{g} \cdot \mathbf{z}\right)}-\frac{1}{3},
$$

with $\mathbf{s}_{g}$, the deviatoric stress tensor $\left(\mathbf{s}_{g}=\sigma_{g}-\operatorname{tr}\left(\boldsymbol{\sigma}_{g}\right) \mathbf{I} / 3\right)$. Let's consider more closely the term $N_{g}=N_{\mathrm{xx}}^{d}+1 / 3$ in order to simplify its expression. In the case of a uniaxial stress of amplitude $\sigma$ applied in the direction parallel to the magnetic field $\mathbf{H}_{g}, N_{g}$ reduces to:

$$
N_{g}=\frac{1}{1+2 \exp (-K \sigma)} .
$$

Following the principles of magneto-elastic equivalent stresses [27], we try to express $N_{g}$, in the general multiaxial case, as a function of a scalar value depending on stress. This scalar value (the equivalent stress $\left.\sigma_{g}^{e q}\right)$ is the uniaxial stress that, applied in the direction parallel to the magnetic field, would lead to the same configuration effect. The equation to solve is:

$$
\frac{1}{1+2 \exp \left(-K \sigma_{g}^{e q}\right)}=\frac{\exp \left(K \mathbf{x} \cdot \mathbf{s}_{g} \cdot \mathbf{x}\right)}{\exp \left(K \mathbf{x} \cdot \mathbf{s}_{g} \cdot \mathbf{x}\right)+\exp \left(K \mathbf{y} \cdot \mathbf{s}_{g} \cdot \mathbf{y}\right)+\exp \left(K \mathbf{z} \cdot \mathbf{s}_{g} \cdot \mathbf{z}\right)} .
$$

A few calculations lead to the following expression of $\sigma_{g}^{e q}$ :

$$
\sigma_{g}^{e q}=\frac{2}{3 A_{s} \lambda_{100}} \ln \left(\frac{2 \exp \left(K \mathbf{x} \cdot \mathbf{s}_{g} \cdot \mathbf{x}\right)}{\exp \left(K \mathbf{y} \cdot \mathbf{s}_{g} \cdot \mathbf{y}\right)+\exp \left(K \mathbf{z}_{\mathbf{s}_{g}} \cdot \mathbf{z}\right)}\right)
$$

Assuming that the quantity $K \mathbf{u} . \mathbf{s}_{g} . \mathbf{u}$ is small compared to 1 (for all $\mathbf{u}$ ), and using a first-order Taylor development of the exponential function, we write:

$$
\begin{aligned}
\exp \left(K \mathbf{y} \cdot \mathbf{s}_{g} \cdot \mathbf{y}\right)+\exp \left(K \mathbf{z}_{\mathbf{s}} \cdot \mathbf{\mathbf { s } _ { g }} \mathbf{z}\right) & \approx 2+K \mathbf{y} \cdot \mathbf{s}_{g} \cdot \mathbf{y}+K \mathbf{z} \cdot \mathbf{s}_{g} \cdot \mathbf{z} \\
& \approx 2-K \mathbf{x} \cdot \mathbf{s}_{g} \cdot \mathbf{x} \quad\left(\text { because } \operatorname{tr}\left(\mathbf{s}_{g}\right)=0\right) \\
& \approx 2 \exp \left(-\frac{1}{2} K \mathbf{x} \cdot \mathbf{s}_{g} \cdot \mathbf{x}\right) .
\end{aligned}
$$

The expression of $\sigma_{g}^{e q}$ then reduces to:

$$
\sigma_{g}^{e q}=\frac{3}{2} \mathbf{x} \cdot \mathbf{s}_{g} \cdot \mathbf{x} .
$$

This expression was already obtained using another approach [20], and was named the deviatoric equivalent stress [27]. 
The expression of $N_{g}$ is finally given by:

$$
N_{g}=\frac{1}{1+2 \exp \left(-K \sigma_{g}^{e q}\right)} .
$$

$K$ is a material parameter defined by $K=\frac{3}{2} A_{s} \lambda_{100}$ in the simplified single-crystal case detailed above. It was shown in [25] that in the case of a polycrystal, $\lambda_{100}$ should be replaced by $\lambda_{m}$ the maximum magnetostriction strain in the absence of magnetisation rotation. As a first approximation, assuming uniform stress within the material, $\lambda_{m}$ can be taken as $\frac{2}{5} \lambda_{100}[12]$ :

$$
K=\frac{3}{2} A_{s} \lambda_{m} \approx \frac{3}{5} A_{s} \lambda_{100}
$$

$\sigma_{g}^{e q}$ is the scalar equivalent stress defined in [20] in order to account for the possible multiaxiality of stress (h being the direction of the magnetic field $\mathbf{H}_{g}$ ):

$$
\sigma_{g}^{e q}=\frac{3}{2} \mathbf{h} \cdot \mathbf{s}_{g} \cdot \mathbf{h} .
$$

The configuration field $\mathbf{H}_{g}^{\text {conf }}$ is then defined as a function of $N_{g}$, with $\eta$ a material parameter:

$$
\mathbf{H}_{g}^{\text {conf }}=\eta\left(N_{g}-\frac{1}{3}\right) \mathbf{M}_{g} .
$$

The coercive field is assumed to show the same dependence to the applied stress, through the parameter $k_{r}$ (defining the coercive field in Hauser's formulation). Indeed, the coercive field is linked to the configuration effect because the probability of encountering pinning centres depends on the space between domain walls. This configuration effect can then rely as well on the schematic illustrations of Figs. 11, 12 and 13. This is the reason why the following expression has been proposed for $k_{r}$ :

$$
k_{r}=k_{r}^{0}\left(1-\left(N_{g}-\frac{1}{3}\right)\right) .
$$

\section{Appendix C: Localisation operators}

Scale transition rules are required to express local loadings (e.g. at the single-crystal scale) as a function of macroscopic loadings (e.g. at the polycrystal scale). The definition of this scale transition is non-trivial and relies on localisation operators. This appendix details the calculation procedure for the localisation operators used in Eqs. (15) and (16).

\section{Appendix C:.1 Mechanical localisation}

The calculation of the localisation tensor $\mathcal{B}_{g}^{\sigma}$ requires several intermediate steps. An inclusion problem is considered first. The Eshelby tensor $\mathcal{N}_{g}$ corresponding to this inclusion problem is calculated [28]. It depends on the shape of the inclusion and on the elastic properties of the infinite medium. The shape of the inclusion is representative for the phase distribution [29]. If the grain distribution is isotropic, a spherical inclusion is chosen. In the case of a self-consistent calculation, the elastic stiffness tensor of the infinite medium is the self-consistent estimate $\tilde{\mathcal{C}}_{m}$. The monograph by Mura [30] provides the guidelines for the practical calculation of the Eshelby tensor. The Hill constraint tensor $\mathcal{C}_{g}^{*}$ is then defined (Eq. (49)), from which the strain localisation tensor $\mathcal{A}_{g}^{\sigma}$ (Eq. (50)) and the stress concentration tensor $\mathcal{B}_{g}^{\sigma}$ are deduced (Eq. (51)). $\mathcal{I}$ is the fourth-order identity tensor. The incompatibility tensor $\mathcal{L}_{g}^{\sigma}$ is finally deduced (Eq. (52)).

$$
\begin{aligned}
\mathcal{C}_{g}^{*} & =\tilde{\mathcal{C}}_{m}:\left(\mathcal{N}_{g}^{-1}-\mathcal{I}\right) . \\
\mathcal{A}_{g}^{\sigma} & =\left(\mathcal{C}_{g}+\mathcal{C}_{g}^{*}\right)^{-1}:\left(\tilde{\mathcal{C}}_{m}+\mathcal{C}_{g}^{*}\right) . \\
\mathcal{B}_{g}^{\sigma} & =\mathcal{C}_{g}: \mathcal{A}_{g}^{\sigma}: \tilde{\mathcal{C}}_{m}^{-1} . \\
\mathcal{L}_{g}^{\sigma} & =\left(\mathcal{C}_{g}^{-1}+\mathcal{C}_{g}^{*-1}\right)^{-1} .
\end{aligned}
$$


Appendix C:.2 Magnetic localisation

The same approach applies for the magnetic field localisation. The depolarising tensor $\mathbf{N}_{g}$ is calculated (see for instance [31] or [32]). The self-consistent estimate $\tilde{\chi}_{m}$ for the magnetic susceptibility is also used. The intermediate tensor $\chi_{g}^{*}$, and localisation operators $\mathcal{A}_{g}^{H}$ and $\mathcal{B}_{g}^{H}$ are then calculated. I is the second-order identity tensor. The incompatibility tensor $\mathcal{M}_{g}^{H}$ is finally deduced (Eq. (56)).

$$
\begin{aligned}
\chi_{g}^{*} & =\tilde{\chi}_{m} \cdot\left(\mathbf{N}_{g}^{-1}-\mathbf{I}\right) . \\
\mathcal{A}_{g}^{H} & =\left(\chi_{g}+\chi_{g}^{*}\right)^{-1} \cdot\left(\tilde{\chi}_{m}+\chi_{g}^{*}\right) . \\
\mathcal{B}_{g}^{H} & =\chi_{g} \cdot \mathcal{A}_{g}^{H} \cdot \tilde{\chi}_{m}^{-1} . \\
\mathcal{M}_{g}^{H} & =\left(\chi_{g}+\chi_{g}^{*}\right)^{-1} .
\end{aligned}
$$

In the context of this paper, the assumptions used in [12] have been used. The local magnetic behaviour is assumed isotropic for the definition of the localisation rules, so that $\mathcal{A}_{g}^{H}$ reduces to 1 and $\mathcal{M}_{g}^{H}$ to $1 /\left(3+2 \tilde{\chi}_{m}\right)$ where $\tilde{\chi}_{m}$ is then a scalar. A detailed explanation for the definition of scale transition rules in the generic case of coupled behaviour can be found in [22].

\section{Appendix D: Guidelines for the identification of material parameters}

The proposed modelling approach makes use of fifteen material parameters to predict the multiaxial dissipative magneto-elastic behaviour of polycrystalline ferro- or ferrimagnetic materials. These parameters are complemented by the crystallographic texture of the material. This appendix aims at providing some guidelines to identify these parameters.

The crystallographic texture is used in the form of a discrete orientation distribution function. It can be directly obtained from EBSD measurements. It can also be extracted from X-ray diffraction patterns.

The parameters describing the single-crystal behaviour are the saturation magnetisation $M_{S}$, the magnetocrystalline anisotropy constants $K_{1}$ and $K_{2}$, the magnetostriction coefficients $\lambda_{100}$ and $\lambda_{111}$ and the elastic coefficients $C_{11}, C_{12}$ and $C_{44}$. These are standard physical constants, their definition is unambiguous and their values can usually be found in Physics textbooks.

The parameter $A_{s}$ has been shown to be proportional to the initial slope of the unstressed anhysteretic magnetisation curve [12]: $A_{s}=\frac{3 \chi^{o}}{\mu_{0} M_{s}^{2}}$. It can then be identified from a low-field anhysteretic measurement under no applied stress.

The parameter $\Sigma^{c}$ controls-together with the crystallographic texture-the macroscopic anisotropy of the material. It can be identified so as to adjust the difference between the maximum magnetostriction of the rolling and transverse directions (see Fig. 5). It can then be identified from two magnetostriction measurements (one along RD, one along TD) at high magnetic field under no applied stress.

$\eta$ allows describing the non-monotonic effect of stress on the magnetic behaviour. It can be identified from a susceptibility measurement under stress (see Fig. 7). The sensitivity to stress is much higher at low field. It is then suggested to perform the identification of $\eta$ from low-field anhysteretic measurements under uniaxial stress. Tension configurations should usually be sufficient for the purpose of this identification.

The last four parameters $k_{r}^{0}, c_{r}, k_{a}$ and $\kappa_{g}^{i n i}$ control the dissipative behaviour according to Hauser's approach [23]. They can be identified so as to adjust the description of a major magnetisation loop under no applied stress starting from a demagnetised state. $k_{r}^{0}$ controls the coercive field amplitude, $c_{r}$ the first magnetisation behaviour and $k_{a}$ and $\kappa_{g}^{i n i}$ the width and inclination of the hysteresis cycle.

As a summary, the material parameters can be identified from anhysteretic measurements at low field under uniaxial stress (tension), from a magnetostriction measurement at high field for two perpendicular directions and from a major hysteresis loop under no applied stress. The model can then be used to predict the material response under any magneto-elastic loading, including multiaxial configurations.

\section{References}

1. Bernard, L., Mininger, X., Daniel, L., Krebs, G., Bouillault, F., Gabsi, M.: Effect of stress on switched reluctance motors: a magneto-elastic finite-element approach based on multiscale constitutive laws. IEEE Trans. Magn. 47(9), 2171-2178 (2011) 
2. Bozorth, R.M.: Ferromagnetism. pp. 595-712. Van Nostrand Company, New York (1951)

3. Cullity, B.D., Graham, C.D.: Introduction to Magnetic Materials. pp. 241-273. Wiley, New Jersey (2011)

4. LoBue, M., Basso, V., Fiorillo, F., Bertotti, G.: Effect of tensile and compressive stress on dynamic loop shapes and power losses of fe-si electrical steels. J. Magn. Magn. Mater. 196(197), 372-374 (1999)

5. Maugin, G.A.: Continuum Mechanics Through the Twentieth Century. Springer, (2013)

6. Maugin, G.A.: The method of virtual power in continuum mechanics: application to coupled fields. Acta Mech. 35, $1-70(1980)$

7. Maugin, G.A.: Continuum Mechanics of Electromagnetic Solids. North-Holland, Amsterdam (1988)

8. Eringen, A.C., Maugin, G.A.: Electrodynamics of Continua. Springer, New York (1990)

9. Armstrong, W.D.: Magnetization and magnetostriction processes in $\mathrm{Tb}(0.27-0.30) \mathrm{Dy}(0.73-0.70) \mathrm{Fe}(1.9-2.0)$. J. Appl. Phys. 81, 2321-2326 (1997)

10. Buiron, N., Hirsinger, L., Billardon, R.: A multiscale model for magneto-elastic couplings. J. Phys. IV 9, 187-196 (1999)

11. Armstrong, W.D.: A directional magnetization potential based model of magnetoelastic hysteresis. J. Appl. Phys. 91, 2202$2210(2002)$

12. Daniel, L., Hubert, O., Buiron, N., Billardon, R.: Reversible magneto-elastic behavior: a multiscale approach. J. Mech. Phys. Solids 56(3), 1018-1042 (2008)

13. Daniel, L., Galopin, N.: A constitutive law for magnetostrictive materials and its application to Terfenol-D single and polycrystals. Eur. Phys. J. Appl. Phys. 42(2), 153-159 (2008)

14. Néel, L.: Les lois de l'aimantation et de la subdivision en domaines élémentaires d'un monocristal de fer. J. Phys. Radiat., 5, 241-251 (1944)

15. Rekik M, Hubert O, Daniel L Influence of a multiaxial stress on the reversible and irreversible magnetic behaviour of a 3\% Si-Fe alloy. Int. J. Appl. Electromagn. Mech. 44(3-4), 301-315 (2014)

16. Motogi, S., Maugin G, A.: Elastic moduli of demagnetized polycrystalline ferromagnets. J. Phys. D:Appl. Phys. 16, 14591467 (1993)

17. du Trémolet de Lacheisseirie, E.: Magnetostriction-Theory and applications of magnetoelasticity. CRC Press, Boca Raton (1993)

18. Hubert, O., Daniel, L.: Effect of plastic straining on magnetostriction of ferromagnetic polycrystals experiments and multiscale modeling. J. Magn. Magn. Mater. 304, 489-491 (2006)

19. Hubert, O., Daniel, L.: Multiscale modeling of the magneto-mechanical behavior of Grain Oriented Silicon Steels. J. Magn. Magn. Mater. 320(7), 1412-1422 (2008)

20. Daniel, L., Hubert, O.: An equivalent stress for the influence of multiaxial stress on the magnetic behavior. J. Appl. Phys. 105(7), 07A313 (2009)

21. Vieille, B., Buiron, N., Pellegrini, Y.P., Billardon, R.: Modelling of the magnetoelastic behaviour of a polycrystalline ferrimagnetic material. J. Phys. IV 115, 129-140 (2004)

22. Corcolle, R., Daniel, L., Bouillault, F.: Generic formalism for homogenization of coupled behaviors :application to magnetoelectroelastic behavior. Phys. Rev. B 78(21), 214110 (2008)

23. Hauser, H.: Energetic model of ferromagnetic hysteresis:Isotropic magnetization. J. Appl. Phys. 96(5), $2753-2767$ (2004)

24. Hubert, O., Daniel, L Billardon, R.: Experimental analysis of the magnetoelastic anisotropy of a non-oriented silicon iron alloy. J. Magn. Magn. Mater. 254, 352-354 (2003)

25. Daniel, L., Hubert, O.: An analytical model for the $\Delta$ E effect in magnetic materials. Eur. Phys. J. Appl. Phys. 45, 31101 (2009)

26. Rizzo, K.J., Hubert, O., Daniel, L.: A multiscale model for piezomagnetic behavior. Eur. J. Electrical Eng. 12(4), 525-540 (2009)

27. Hubert, O., Daniel, L.: Energetical and multiscale approaches for the definition of an equivalent stress for magneto-elastic couplings. J. Magn. Magn. Mater. 323(13), 1766-1781 (2011)

28. Eshelby J, D.: The determination of the elastic field of an ellipsoidal inclusion, and related problems. Proc. R. Soc. Lond. A 421, 376-396 (1957)

29. Bornert M, Bretheau T, Gilormini P Homogénéisation en mécanique des matériaux. Tome 1 : Matériaux aléatoires élastiques et milieux périodiques. Hermès Science (2001)

30. Mura, T.: Micromechanics of Defects in Solids. Martinus Nijhoff Publishers, Dordrecht, MA (1982)

31. Sihvola A Electromagnetic Mixing Formulas and Applications. IEE Electromagnetic Waves Series 47 (1999)

32. Milton, G.W.: The Theory of Composites. Cambridge University Press, New York (2002) 